\title{
Bond Strength and Microleakage of a Novel Glass Ionomer Cement Containing Silver Diamine Fluoride
}

\author{
Prim Auychai ${ }^{10}$ Nichakorn Khumtrakoon ${ }^{2}$ Chonticha jitongart ${ }^{2}$ Punnamas Daomanee ${ }^{2}$ \\ Arunee Laiteerapong ${ }^{3}$
}

${ }^{1}$ Department of Pediatric Dentistry, Faculty of Dentistry, Chulalongkorn University, Bangkok, Thailand

${ }^{2}$ CU Dental Innovation Center, Faculty of Dentistry, Chulalongkorn University, Bangkok, Thailand

${ }^{3}$ Faculty of Dentistry, Chulalongkorn University, Bangkok, Thailand Eur J Dent 2022;16:606-611.
Address for correspondence Prim Auychai, DDS, MS, Department of Pediatric Dentistry, Faculty of Dentistry, Chulalongkorn University, Bangkok 10330, Thailand (e-mail: prim.a@chula.ac.th).

\begin{abstract}
Keywords

- glass ionomer cement

- microleakage

- shear bond strength

- silver diamine fluoride

Objectives To investigate the shear bond strength and microleakage of glass ionomer cement (GIC) containing silver diamine fluoride (SDF).

Materials and Methods Sound human permanent premolars were divided into the following three groups: 1) GIC (Fuji IX), 2) GICSDF-S: GIC+SDF (Saforide), and 3) GICSDF-T: GIC + SDF (Topamine). Shear bond strength ( $n=14 /$ group) was measured using a universal testing machine and compared between groups (one-way ANOVA and Tukey HSD, $p<0.05$ ). Microleakage ( $n=15 /$ group) at enamel and dentin margins was scored using a stereomicroscope (10x) and compared between groups (Chi-square, $p<0.05)$.

Results There were significant differences in shear bond strength between the GIC and GICSDF-S groups and between the GIC and GICSDF-T groups. The GIC group had the lowest shear bond strength among the groups; however, there was no significant difference between the GICSDF-S and GICSDF-T groups. The microleakage test results were not significantly different between groups at the enamel margin or dentin margins. Although the GIC group demonstrated a higher dye penetration score at the enamel and dentin margins, the difference was not significant.

Conclusions Within the limitations of this study, we conclude that incorporating SDF into $\mathrm{GIC}$ results in higher shear bond strength while not increasing microleakage at the enamel and dentin margins.
\end{abstract}

\section{Introduction}

Dental caries is the most common chronic childhood disease, and its prevalence has increased among children of 2 to 5 years of age worldwide, making this population a global priority for action. ${ }^{1,2}$ Untreated carious lesions can lead to toothache, pain, and infection. These consequences affect

published online December 17, 2021
DOI https://doi.org/ 10.1055/s-0041-1736329. ISSN 1305-7456. children's oral health and their general health, such as their growth, cognitive development, and quality of life. ${ }^{3}$

Although dental caries can be treated by conventional surgical interventions, not all affected groups have access to dental care, especially vulnerable groups such as young children in rural areas. The atraumatic restorative treatment

\section{(c) 2021. The Author(s).}

This is an open access article published by Thieme under the terms of the Creative Commons Attribution License, permitting unrestricted use, distribution, and reproduction so long as the original work is properly cited. (https://creativecommons.org/licenses/by/4.0/)

Thieme Medical and Scientific Publishers Pvt. Ltd., A-12, 2nd Floor, Sector 2, Noida-201301 UP, India 
(ART) technique is an alternative approach for managing dental caries, which involves removing the carious tissue using only hand instruments, followed by applying an adhesive material. ${ }^{4}$ The restorative material of choice for ART is a high-viscosity glass ionomer cement (GIC). ${ }^{5}$ GIC provides biocompatibility, chemical adhesion to the tooth surface, a coefficient of thermal expansion similar to that of natural teeth, and fluoride release. However, a major concern of this technique is the residual cariogenic bacteria that remain under the restorations. ${ }^{6}$ To overcome this concern, multiple studies have investigated GICs modified by incorporating various antimicrobial agents (e.g. chlorhexidine, antibiotics, and propolis) to improve the antimicrobial property, however, the physical properties, that is, compressive strength and setting time of GIC were compromised. ${ }^{7-11}$

Silver diamine fluoride (SDF), a widely used antibacterial compound, was introduced in 1969 by Nishino et al and has been used to stop caries progression, due to its antimicrobial effect, and enhance remineralization due to its fluoride content. $^{12}$ Multiple published systematic and updated reviews indicated that SDF application successfully arrests dental caries in children, ${ }^{13,14}$ and a recent report found that SDF does not adversely affect the bond strength between GIC and carious dentin of primary teeth. ${ }^{15}$ A previous study from our group investigating the effect of incorporating 38\% SDF at different concentrations to improve the antibacterial activity of GIC demonstrated that GIC containing SDF at 5\% (v/v GICliquid) and $0.0152 \mathrm{~g} \mathrm{SDF} / \mathrm{mL}$ had a higher antibacterial effect compared with GIC alone and met the International Organization for Standardization (ISO) standards for setting time and compressive strength without affecting the GIC fluoride release pattern compared with that of conventional GIC. ${ }^{16}$

This novel GIC maximized the effect of fluoride release from GIC in preventing carious lesions and improved its antibacterial and remineralization effects. ${ }^{16}$ However, the bonding efficacy and microleakage of GIC-containing SDF (GICSDF) have not been investigated. Therefore, the purpose of this study was to investigate the shear bond strength of SDF-GIC to dentin and its microleakage in cavities in extracted teeth.

\section{Materials and Methods}

This study followed the Checklist for Reporting In vitro Study (CRIS) guidelines for in vitro studies as discussed in the 2014 concept note. $^{17}$

A conventional GIC (Fuji IX) was used as control (GIC). Experimental GICs (GICSDF-S and GICSDF-T) were prepared by incorporating two brands of $38 \%$ SDF, Saforide and Topamine, into the liquid portion of the GIC ( $2 \mu \mathrm{L}$ each) and handmixed with the GIC powder $(\mathrm{P} / \mathrm{L}=3.4: 1[\mathrm{w} / \mathrm{w}])$.

Eighty-seven extracted human premolars were collected for measuring the bond strength and microleakage. The study protocol was approved by the Human Research Ethics Committee (HREC-DCU 2018-045).

The GICSDF bonding efficacy and microleakage assays were performed, according to ISO/TS 11405 Dentistry Testing for dental adhesion to tooth structure. ${ }^{18}$ Forty-two and 45 sound human premolars were used for the shear bond strength measurement and microleakage test, respectively. The teeth were washed under running water, blood and remaining adherents removed, and stored in $10 \%$ thymol in distilled water at $23 \pm 2{ }^{\circ} \mathrm{C}$ for not more than 1 month after extraction until used. The experimental groups were as follows: 1) GIC (Fuji IX, GC, Tokyo, Japan), 2) GICSDF-S (Fuji IX + SDF [Saforide, Toyo Seiyaku Kasei Co. Ltd., Osaka, Japan]), and 3) GICSDF-T (Fuji IX + SDF [Topamine, Dentalife Australia Pty. Ltd., Ringwood, Australia]).

\section{Shear Bond Strength Measurement}

Forty-two of the 87 teeth were mounted in cold-curing resin and ground flat using a conventional model trimmer with water to expose the dentin. The specimens were randomly divided into three groups $(n=14)$. A silicone mold was used as a bonding template and was attached to the dentin surface. Dentin conditioner (GC Corp., Tokyo, Japan) was applied to the specimen surface for 10 seconds using a cotton pellet, rinsed off with water spray, the surface gently airdried, and restored with handmixed GIC.

The GIC powder and liquid were handmixed as described above at $23 \pm 2{ }^{\circ} \mathrm{C}$ and $50 \pm 5 \%$ relative humidity and placed in a silicone mold. After setting, the mold was gently removed and the surfaces were coated with GC, stored in water at $37 \pm 2{ }^{\circ} \mathrm{C}$ for 24 hours, and mounted in a universal testing machine (EZ-s; Shimadzu Corporation, Kyoto, Japan) such that the adhesive interface of the specimen was fixed within $0.5 \mathrm{~mm}$ of the shearing blade ( $\mathbf{- F i g}$. 1A). The specimen was sheared at a load rate of $50 \pm 2 \mathrm{~N} / \mathrm{min}$ until failure. The bond strength data were obtained in Newtons, and converted to megapascals.

\section{Microleakage Test}

Forty-five of the 87 teeth were used for the microleakage test and randomly divided into three groups $(n=15)$. The teeth were stored in distilled water at $23 \pm 2{ }^{\circ} \mathrm{C}$ at least 12 hours before use. A class $\mathrm{V}$ cavity $3.0 \pm 0.1 \mathrm{~mm}$ in diameter, approximately $1.5-\mathrm{mm}$ deep, with a cavosurface angle of approximately $90^{\circ}$, was prepared using a high-speed cylindrical diamond burr with waterspray at the midbuccal surface of each tooth, with the occlusal margin in enamel and the gingival margin in dentin. The cavity walls were finished with a straight flat fissure carbide burr with flat end without cross-cuts. The mixed GIC was placed in the cavity, the specimens were immersed in water immediately, stored at $23 \pm 2{ }^{\circ} \mathrm{C}$ for 24 hours, and thermocycled for 500 cycles (20 seconds in water at $5{ }^{\circ} \mathrm{C}$ and $55^{\circ} \mathrm{C}$ each, 5 seconds transfer time). The specimens were immersed in methylene blue solution for 10 minutes and cut longitudinally at each side of the cavity midline, using a slow-speed diamond saw (ISOMET $^{\mathrm{TM}} 1000$, Buehler, Illinois, USA) under watercooling, generating three sections from each tooth. The sections were examined by two examiners, with the help of a stereomicroscope (SZ 61; Olympus Corporation, Tokyo, Japan) (10x), for dye penetration along the cavity walls using the scale described in ISO TR11405 (-Fig. 1B and - Table 1). The examiners randomly reevaluated $20 \%$ of the specimens to assess the inter- and intraexaminer reliability. 

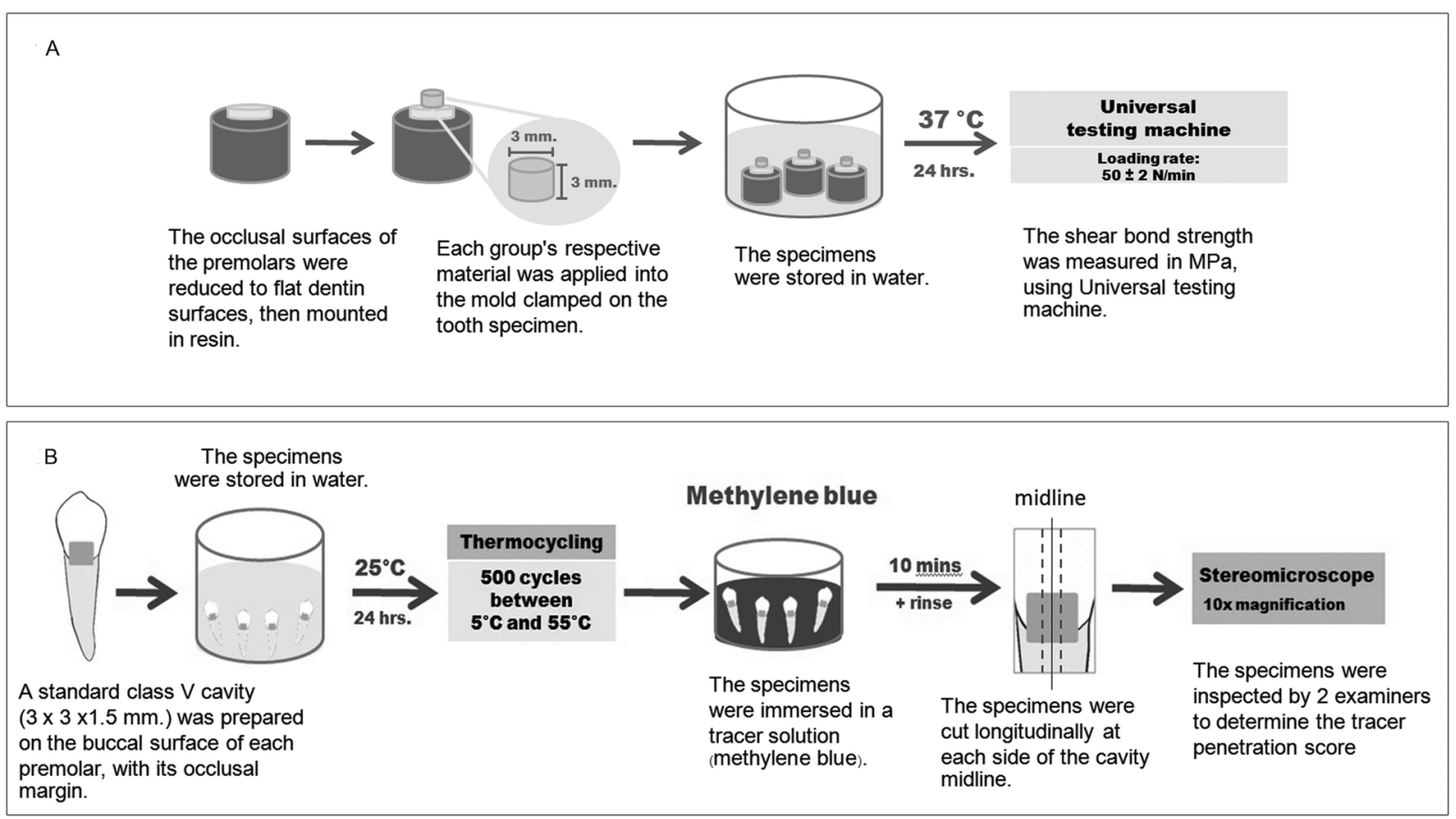

Fig. 1 (A) Flowchart of the shear bond strength test, (B) flowchart of microleakage test.

\section{Statistical Analysis}

The data was collected, tabulated, and statistically analyzed using SPSS version 21 statistical analysis package software (SPSS Inc., Chicago, IL, USA). The shear bond strength values (MPa) were analyzed using one-way ANOVA and Tukey HSD. Statistical significance was considered at $p<0.05$. The enamel and dentin microleakage scores of each group of restorations were analyzed using the Chi-square test with statistical significance at $p<0.05$. Kappa statistics was used to determine the interexaminer and intraexaminer reliability.

\section{Results}

\section{Shear Bond Strength Test}

The control group samples (GIC) had a mean shear bond strength and standard deviation $( \pm \mathrm{SD})$ of $(3.87 \pm 1.96) \mathrm{MPa}$, while the test groups (GICSDF-S and GICSDF-T) had a mean shear bond strengths of $(7.09 \pm 2.31)$ and $(7.79 \pm 3.15) \mathrm{MPa}$, respectively (-Fig. 2 ). The results of one-way ANOVA and Tukey HSD tests indicated that there were significant differ- ences in shear bond strength between the GIC and GICSDF-S groups and between the GIC and GICSDF-T groups. The GIC group had the lowest shear bond strength among the groups; however, there was no significant difference between the GICSDF-S and GICSDF-T groups.

\section{Microleakage Test}

Kappa analysis indicated that the interexaminer reliability in scoring was 1.00 , which indicated perfect agreement. The intraexaminer reliability scores were 0.97 and 0.88 for each examiner, respectively, which indicated near perfect agreement. $^{19}$

The microleakage results of all groups in enamel and dentin are presented in -Fig. 3. At the enamel margins, the groups demonstrated a similar leakage pattern, with the majority having a 0 score (no leakage). The Chi-square test indicated no significant differences between the groups $(p>0.05)$ ( - Fig. 3A). At the dentin margins, the GIC group tended to have a higher dye penetration score than the GICSDF-S and GICSDF-T groups (-Fig. 3B); however, the difference was not significant.

Table 1 Quantification of tracer along the cavity wall at enamel margins and dentin margins

\begin{tabular}{|l|l|l|}
\hline Score & Quality of tracer penetration at enamel margins & Quality of tracer penetration at dentin margins \\
\hline 0 & No penetration & No penetration \\
\hline 1 & Penetration into the enamel part of the cavity wall & $\begin{array}{l}\text { Penetration into dentin/material interface but not } \\
\text { including the pulpal floor of the cavity }\end{array}$ \\
\hline 2 & $\begin{array}{l}\text { Penetration into dentin/material interface but not } \\
\text { including the pulpal floor of the cavity }\end{array}$ & Penetration including the pulpal floor of the cavity \\
\hline 3 & Penetration including the pulpal floor of the cavity & - \\
\hline
\end{tabular}




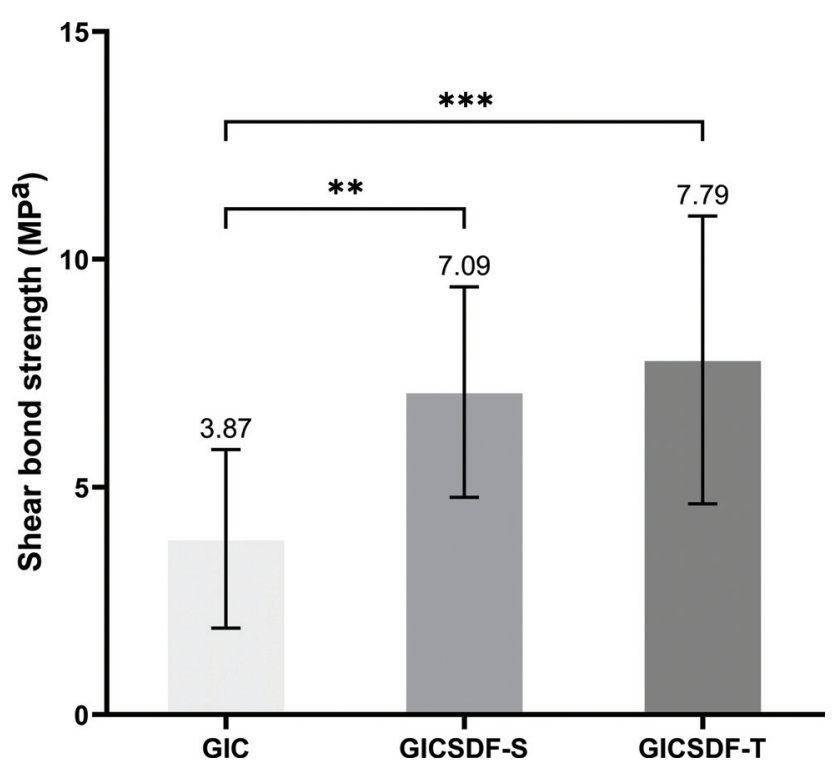

Fig. 2 Mean shear bond strength and standard deviation (SD) of the different groups tested (using one-way ANOVA and multiple comparisons by Tukey HSD tests). GIC = glass ionomer cement; GICSDF-S = Fuji IX + SDF (Saforide); GICSDF-T = Fuji IX + SDF (Topamine). ${ }^{* *} p<0.01^{* * *} p<0.001$.
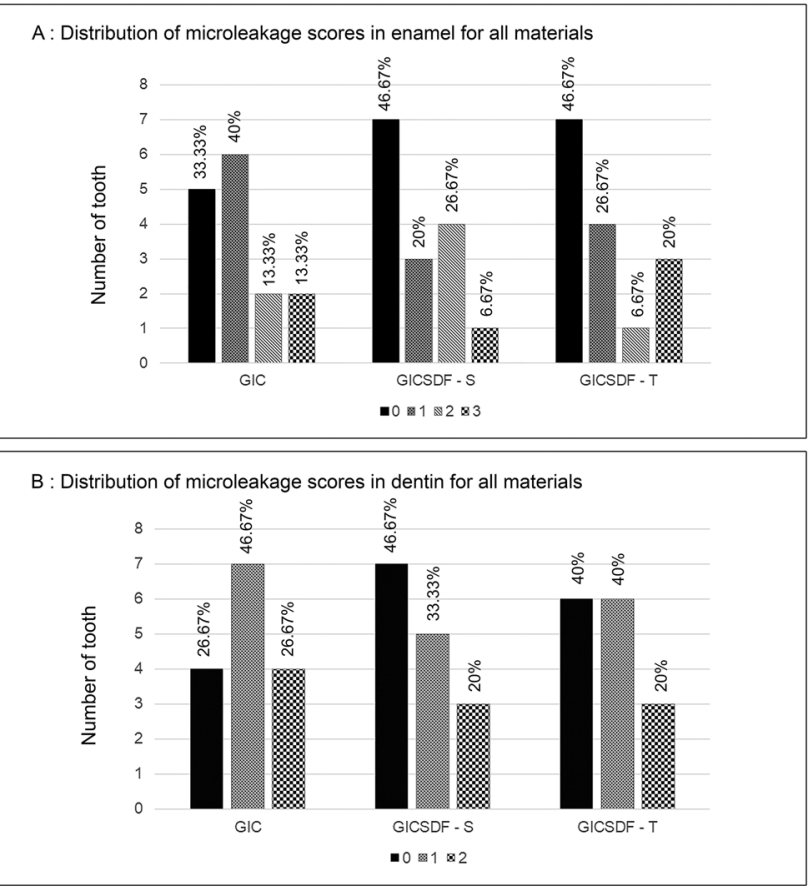

Fig. 3 (A) Number of surfaces with leakage at the enamel margins, (B) Number of surfaces with leakage at the dentin margins (using the Chi-square test with statistical significance at $p<0.05) . \mathrm{GIC}=$ glass ionomer cement; GICSDF-S = Fuji IX + SDF (Saforide); GICSDF-T = Fuji IX + SDF (Topamine).

\section{Discussion}

The objectives of this study were to investigate if adding SDF to GIC would affect its bond strength and microleakage. The results demonstrated that the shear bond strength of the GIC containing SDF was improved and that the microleakage was not adversely affected.

The present study used the shear bond strength and microleakage tests, according to the ISO/IS 11405. This ISO was the standard at the time this study was conducted. However, this has been withdrawn and is currently undergoing revision. Bonding efficacy is an important property of dental materials, and the shear bond strength test is used for defining bonding efficacy, because it is less technique sensitive and emphasizes the strength at the bonded interface compared with other bonding efficacy tests. ${ }^{20}$ The microleakage test is used to investigate the cavity-sealing property of a material and can be assessed by several methods, including using dyes, chemical tracers, radioactive tracers, scanning electron microscopy, neutron activation analysis, and fluid infiltration. In this study, the dye leakage method was used, because it is simple, inexpensive, fast, and the use of complex laboratory equipment is not required. ${ }^{21}$ We performed 500 cycles of thermocycling to simulate the temperature changes of the oral environment. This number of thermocycles has been shown in a prior study to be equivalent to approximately 1 month of clinical service. ${ }^{22}$

In this study, we used two commercial 38\% SDF solutions that contain $44,800 \mathrm{ppm}$ fluoride, that is, Saforide and Topamine, to add to the GIC and investigated the material properties. Saforide is a well-known SDF product; however, Topamine is the only brand available in Thailand. We found that the shear bond strength was improved in the experimental groups compared with the control group, and microleakage was not increased.

The adhesion of dental materials to tooth surface has been extensively investigated. GICs bond to the tooth surface by ionic bonds being formed between the carboxylate groups on the polyacid molecules and calcium ions in the tooth surface. $^{23}$ Dentin is usually associated with a lower bond strength compared with enamel, due to dentin having a greater proportion of water and organic materials. Previous studies have shown that the typical shear bond strengths of GICs to dentin ranged from 1 to $3 \mathrm{MPa}$ and rarely exceed $5 \mathrm{MPa}{ }^{24,25}$ The shear bond strength result of GIC to dentin in our study was $3.68 \pm 1.92 \mathrm{MPa}$, which was similar to other studies. For GIC-containing SDF, we found that the mean shear bond strengths in the GICSDF-S and GICSDF-T were significantly higher compared with the GIC-alone group, while the mean shear bond strengths in the two SDF groups were similar.

In two recent systematic reviews of the influence of SDF application on the bond strength of dentin to various adhesives and to GICs, it was found that although previous applications of SDF had no adverse impact on the bond strength of the GIC, it compromises the bonding of adhesive systems. ${ }^{26,27}$ In the present study, SDF was incorporated into the liquid part of GIC; thus, the result may be different when applying SDF prior to the GIC. Currently, there is no direct evidence of the mechanism of action when adding SDF to GIC.

SDF application can arrest active caries and prevent the development of dental complications; however, there are 
limitations with SDF treatment. The black stain on the SDFarrested caries lesions may cause esthetic concerns, and the chewing function of the cavitated teeth may not be improved because the tooth cavities are not restored. GIC can mask the stained carious lesion associated with SDF, while SDF can minimize the micro/nanoleakage and secondary caries associated with GICs.

SDF can be applied prior to the restorative treatment. However, the lesion cannot be restored with GIC at the same visit. Incorporating SDF into GIC during the restorative procedure would eliminate the need for this extra visit. Furthermore, handmixing was performed because the SDF was incorporated into GIC liquid and cannot be mixed using a mixer. To incorporate SDF into the encapsulated GIC liquid portion would need to be done at the manufacturer level, which was not practical for this initial study. The results of our study indicated that SDF-GIC restorations significantly improved the bond strengths.

Microleakage results in the passage of bacteria, fluids, molecules, or ions between a cavity wall and the restorative material applied to it. ${ }^{28}$ Microleakage between the restoration and the tooth interface can result in the occurrence of postoperative pain, discoloration of the cavity edges, secondary caries, and pulpal inflammation, resulting in postoperative failure.

We used $2 \%$ methylene blue dye in our study because of its ease of manipulation, convenience, low-cost, and the lowmolecular weight of the dye, which is smaller than bacteria and can detect leakage where bacteria could not penetrate. The results of the present study are in agreement with the previous studies that more leakage is seen from the dentin than the enamel margins. ${ }^{29-31}$ The restorations placed without any conditioning also showed significantly greater microleakage. ${ }^{29}$ We used a dentin conditioner, as recommended by the manufacturer.

No statistical difference in microleakage was detected at the enamel or dentin margins between the control and experimental groups; however, a trend toward higher microleakage of GIC compared with GIC-SDF was found. The present findings suggest that the novel glass ionomer and the conventional material have comparable sealing ability.

Because our study was short-term in nature, a long-term clinical trial of GIC-containing SDF is necessary to assess its intraoral performance. Based on the differences in the crystal orientation between permanent and primary teeth, a study on primary teeth should be performed.

\section{Conclusions}

Based on this study's results, the following conclusions can be made: Incorporating SDF into GIC resulted in a higher shear bond strength compared with GIC without SDF and did not adversely affect the microleakage at the enamel and dentin cavity margins; GIC-containing SDF could be a potential restorative material; however, a long-term clinical trial of GIC-containing SDF to assess its intra-oral performance should be performed.

\section{Funding}

The study was financed by a research grant by the Faculty of Dentistry, Chulalongkorn University.

\section{Conflict of Interest}

None declared.

\section{Acknowledgments}

The authors are grateful to Assistant Professor Soranun Chantarangsu for her statistical advice, Dr. Kevin Tompkins, Associate Professor Panida Thanyasrisung and Professor Martin Tyas (University of Melbourne) for manuscript revision.

\section{References}

1 Peres MA, Macpherson LMD, Weyant RJ, et al. Oral diseases: a global public health challenge. Lancet 2019;394(10194):249-260

2 Pitts NB, Zero DT, Marsh PD, et al. Dental caries. Nat Rev Dis Primers 2017;3(03):17030

3 FDI World Dental Association. The Oral Health Atlas. 2nd ed. Accessed August 12, 2020 at: http://www.fdiworldental.org/media/77552/complete_oh_atlas.pdf

4 Frencken JE, Leal SC, Navarro MF. Twenty-five-year atraumatic restorative treatment (ART) approach: a comprehensive overview. Clin Oral Investig 2012;16(05):1337-1346

5 Smales RJ, Yip HK. The atraumatic restorative treatment (ART) approach for primary teeth: review of literature. Pediatr Dent 2000;22(04):294-298

6 Weerheijm KL, Kreulen CM, de Soet JJ, Groen HJ, van Amerongen WE. Bacterial counts in carious dentine under restorations: 2-year in vivo effects. Caries Res 1999;33(02):130-134

7 Frencken JE, Imazato S, Toi C, et al. Antibacterial effect of chlorhexidine- containing glass ionomer cement in vivo: a pilot study. Caries Res 2007;41(02):102-107

8 Yesilyurt C, Er K, Tasdemir T, Buruk K, Celik D. Antibacterial activity and physical properties of glass-ionomer cements containing antibiotics. Oper Dent 2009;34(01):18-23

9 de Castilho AR, Duque C, Negrini TdeC, et al. In vitro and in vivo investigation of the biological and mechanical behaviour of resinmodified glass-ionomer cement containing chlorhexidine. J Dent 2013;41(02):155-163

10 Hafshejani TM, Zamanian A, Venugopal JR, et al. Antibacterial glass-ionomer cement restorative materials: A critical review on the current status of extended release formulations. J Control Release 2017;262:317-328

11 Meneses IHC, Sampaio GAM, Carvalho FG, et al. In vivo biocompatibility, mechanical, and antibacterial properties of cements modified with propolis in different concentrations. Eur J Dent 2020;14(01):77-84

12 Nishino M, Yoshida S, Sobue S, Kato J, Nishida M. Effect of topically applied ammoniacal silver fluoride on dental caries in children. J Osaka Univ Dent Sch 1969;9:149-155

13 Gao SS, Zhao IS, Hiraishi N, et al. Clinical trials of silver diamine fluoride in arresting caries among children: a systematic review. JDR Clin Trans Res 2016;1(03):201-210

14 Crystal YO, Niederman R. Evidence-based dentistry update on silver diamine fluoride. Dent Clin North Am 2019;63(01): 45-68

15 Puwanawiroj A, Trairatvorakul C, Dasanayake AP, Auychai P. Microtensile bond strength between glass ionomer cement and silver diamine fluoride-treated carious primary dentin. Pediatr Dent 2018;40(04):291-295

16 Puangphimolkij T, Jariyamana C, Teeranuwat P, et al. Antibacterial activity, physical properties and fluoride release of a glass 
ionomer cement containing silver diamine fluoride: abstract number SEA0174. Accessed September 10, 2021 at: https:// iadr.abstractarchives.com/abstract/sea-iadr2018-3006784/ development-of-a-novel-glass-ionomer-cement-containingsilver-diamine-fluoride

17 Krithikadatta J, Gopikrishna V, Datta M. CRIS Guidelines (Checklist for Reporting In-vitro Studies): A concept note on the need for standardized guidelines for improving quality and transparency in reporting in-vitro studies in experimental dental research. J Conserv Dent 2014;17(04):301-304

18 ISO/TS 11405:2003 Dental materials-Testing of adhesion to tooth structure. Accessed September 10, 2020 at: https://www. iso.org/standard/31486.html

19 Landis JR, Koch GG. The measurement of observer agreement for categorical data. Biometrics 1977;33(01):159-174

20 Nujella BPS, Choudary MT, Reddy SP, Kumar MK, Gopal T. Comparison of shear bond strength of aesthetic restorative materials. Contemp Clin Dent 2012;3(01):22-26

21 Bogra P, Gupta S, Kumar S. Comparative evaluation of microleakage in class II cavities restored with Ceram X and Filtek P-90: An in vitro study. Contemp Clin Dent 2012;3(01):9-14

22 Gale MS, Darvell BW. Thermal cycling procedures for laboratory testing of dental restorations. J Dent 1999;27(02):89-99

23 Powis DR, Follerås T, Merson SA, Wilson AD. Improved adhesion of a glass ionomer cement to dentin and enamel. J Dent Res 1982;61 (12):1416-1422
24 Poggio C, Beltrami R, Scribante A, Colombo M, Lombardini M. Effects of dentin surface treatments on shear bond strength of glass-ionomer cements. Ann Stomatol (Roma) 2014;5(01):15-22

25 Burke FM, Lynch E. Glass polyalkenoate bond strength to dentine after chemomechanical caries removal. J Dent 1994;22(05):283-291

26 Fröhlich TT, Rocha RO, Botton G. Does previous application of silver diammine fluoride influence the bond strength of glass ionomer cement and adhesive systems to dentin? Systematic review and meta-analysis. Int J Paediatr Dent 2020;30(01): 85-95

27 Jiang $\mathrm{M}$, Mei ML, Wong $\mathrm{M}$, Chu $\mathrm{CH}$, Lo E. Influence of silver diamine fluoride treatment on the microtensile bond strength of glass ionomer cement to sound and carious dentin. Oper Dent 2020;45(05):E271-E279

28 Kidd EA. Microleakage: a review. J Dent 1976;4(05):199-206

29 Delmé KI, Deman PJ, De Bruyne MA, De Moor RJ. Microleakage of four different restorative glass ionomer formulations in class $\mathrm{V}$ cavities: Er:YAG laser versus conventional preparation. Photomed Laser Surg 2008;26(06):541-549

30 Gorseta K, Glavina D, Skrinjaric I. Influence of ultrasonic excitation and heat application on the microleakage of glass ionomer cements. Aust Dent J 2012;57(04):453-457

31 Pontes DG, Guedes-Neto MV, Cabral MF, Cohen-Carneiro F. Microleakage evaluation of class $\mathrm{V}$ restorations with conventional and resin-modified glass ionomer cements. Oral Health Dent Manag 2014;13(03):642-646 